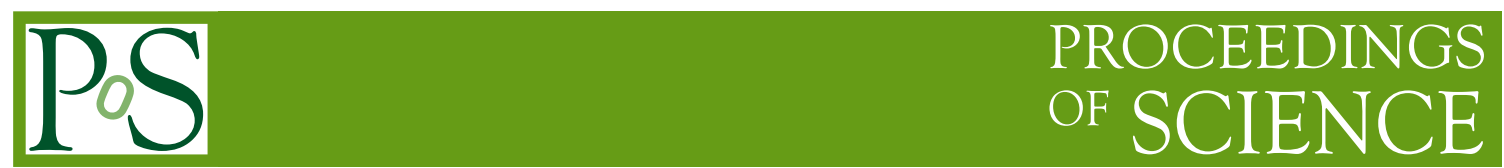

\title{
MPGD-based counters of single photons for Cherenkov imaging counters.
}

\author{
M.Alexeev $^{a}$, R.Birsa ${ }^{a}$, M. Bodlak ${ }^{h}$, F.Bradamante $^{b}$, A.Bressan $^{b}$, M.Büchele $^{c}$, \\ M.Chiosso $^{d}$, P.Ciliberti $^{b}$, S.Dalla Torre ${ }^{a}$, S.Dasgupta $^{a}{ }^{*}$, O.Denisov $^{e}$, V.Duic $^{b}$, \\ M.Finger $^{f}$, M.Finger $\mathbf{J r}^{f}$, H.Fischer $^{c}$, M.Giorgi ${ }^{b}$, B.Gobbo $^{a}$, M.Gregori ${ }^{a}$, F.Herrmann $^{c}$, \\ K.Königsmann $^{c}$, S.Levorato ${ }^{b \dagger}$, A.Maggiora $^{e}$, A.Martin $^{b}$, N. Makke ${ }^{b}$, G.Menon $^{a}$, \\ K.Novakova $^{g}$ J.Novy $^{h}$, D.Panzieri ${ }^{i}$, F.A.Pereira ${ }^{j}$, C.A.Santos ${ }^{j}$, G.Sbrizzai $^{b}$, \\ P.Schiavon $^{b}$, S.Schopferer $^{c}$, M.Slunecka $^{f}{ }$ F.Sozzi $^{a}{ }$ L.Steiger $^{g}$, M.Sulc $^{k}$, \\ F.Tessarotto $^{a}$, J.F.C.A.Veloso ${ }^{j}$ \\ ${ }^{a}$ INFN, Sezione di Trieste, Trieste, Italy \\ ${ }^{b}$ INFN, Sezione di Trieste and University of Trieste, Trieste, Italy \\ ${ }^{c}$ Universität Freiburg, Physikalisches Institut, Freiburg, Germany \\ ${ }^{d}$ INFN, Sezione di Torino and University of Torino, Torino, Italy \\ ${ }^{e}$ INFN, Sezione di Torino, Torino, Italy \\ ${ }^{f}$ Charles University, Prague, Czech Republic and JINR, Dubna, Russia \\ ${ }^{g}$ Research Centre for Special Optics and Optoelectronic Systems (TOPTEC), Institute of Plasma \\ Physics, Academy of Sciences of the Czech Republic, Turnov, Czech Republic \\ ${ }^{h}$ INFN, Czech Technical University, Prague, Czech Republic \\ ${ }^{i} I N F N$, Sezione di Torino and University of East Piemonte, Alessandria, Italy \\ ${ }^{j}$ I3N - Physics Department, University of Aveiro, Aveiro, Portugal \\ ${ }^{k}$ Technical University of Liberec, Liberec, Czech Republic \\ E-mail stefano.levorato@ts.infn.it
}




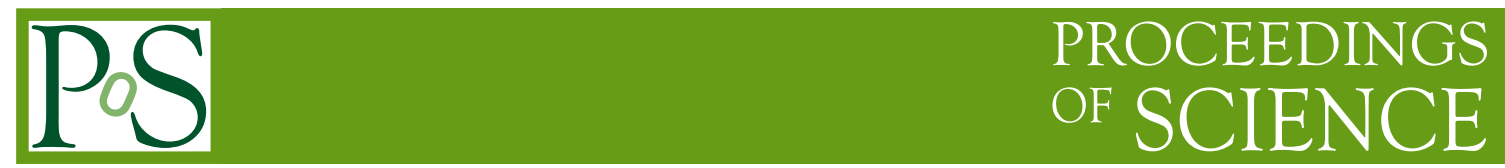

Architectures based on MicroPattern Gas Detectors (MPGD) represent a possible answer to the quest for novel gaseous counters with single photon detection capability able to overcome all the limitations of the present generation of gaseous photon detectors. In Cherenkov imaging counters, gaseous photon detectors are still the unique option when insensitivity to magnetic field, low material budget, and affordable costs in view of large detection surfaces are required. A systematic $R \& D$ programme has been performed for several years to develop novel gas photon detectors base on an arrangement of multiple layers of THick-Gas Electron Multipliers (THGEM): a deep understanding of the THGEM characteristics has been achieved and their parameters have been optimised in view of the photon detection application. Large gains are required to detect effectively single photoelectrons and, after the optimisation process, the novel detectors exhibit electrical stability up to gains as high as to $1-2 \times 10^{5}$ also in presence of radioactive backgrounds. The delicate aspect of the photo electron extraction from a GEM-like photocathode has been studied in detail and conditions for effective extraction have been obtained. The suppression of the signal produced by ionising particles crossing the photon detectors has been proven. In parallel with establishing the detector principle, the engineering towards large-size counters is ongoing and an intermediate size detector with $300 \times 300 \mathrm{~mm}^{2}$ active surface has been successfully operated. Recently a new hybrid approach has been considered: an architecture where the last multiplication stage is obtained by using a MicroMegas arrangement. The completed R\&D studies and the engineering aspects are summarised and the characterization of the hybrid detector prototypes are reported.

Technology and Instrumentation in Particle Physics 2014,

2-6 June, 2014

Amsterdam, the Netherlands

*on leave from Matrivani Institute of Experimental Research and Education, Kolkata

†Corresponding author. 


\section{Introduction}

The future accelerator facilities will be dedicated to the precision determination of the standard model parameter, the constraints of the potential new physics contributions and the direct studies for new physics search. These goals require high precision measurements at high luminosity: this imposes a novel generation of detectors exhibiting unprecedented fine space and time resolution and high rate capabilities. The Higgs discovery with the multi-purpose ATLAS and CMS detectors at LHC became possible at the price of major detector instrumentation progress and pushed most of the technologies at their performance limits. Still open questions remain as the precise determination of the Higgs boson couplings and of the evidence of new particles at LHC. The incoming high luminosity LHC Runs as well as the increased track density at the $14 \mathrm{TeV}$ energy pose severe limitation to the present detectors technology.

Particle Identification also plays nowadays an irreplaceable role in particle physics experiments, as documented by its wide present use. It is also a required ingredient foreseen for the next generation of experiments in a large variety of fields in nuclear and particle physics: quark spectroscopy, K-physics, B-physics, Nucleon structure, heavy ion physics, q-g fusion, hadrons in nuclear matter. In this contest the Ring Imaging Cherenkov technique represents the most powerful approach when wide momenta and large phase space coverage are needed. Photosensitive surfaces of several square meters, needed at the future experiments, can be instrumented only with gaseous photon detectors and the present generation of large size gas photon detectors exhibits performance limitations that dictate a critic review on their future use. On the other side the use of Micro Pattern Gaseous Detectors (MPGDs) sensitive to the single photon can represent a valid alternative option.

\section{MPGD architectures.}

MPGDs are a relatively novel family of particle detector, based on gaseous multiplication using micro pattern electrode structures instead of thin wires as in Multi Wire Proportional Chambers (MWPC). By the use of reduced pitch size of the order around one hundred $\mu \mathrm{m}$, namely an order of magnitude better in granularity respect to wire chambers, these detectors can offer an intrinsic high rate capability, larger than $10^{6} \mathrm{~Hz} / \mathrm{mm}^{2}$, very fine space resolution better than $100 \mu \mathrm{m}$ and time resolutions in the few ns range. The MSGC, introduced in 1988 by Oed[1], was the first micro-structured gas detector; in its first version, it was affected by performance limitations and it came to an effective maturity only some ten years later by introducing technological improvements. The technological progress in the photo lithographic techniques during the 90ies gave rise to extremely powerful devices as the Gas Electron Multiplier (GEM) by Sauli in 1997[2, 3], and the MICRO-MEsh GAseous Structure (MicroMegas) of Giomataris in 1996[4]. These devices show improved operational stability and increased radiation hardness. From these initial ideas, many MPGD architectures have derived such as the thick GEM (THGEM) [5], the REsistive Thick GEM (RETHGEM)[6], the Cobra[7] and the micropixel gas chamber ( $\mu$-PIC).

THGEMs are derived from GEM changing material and using the production procedure of standard PCB technology: mechanical drilling of 0.3-1 mm diameter holes, possibly etched to create a clearance region around the hole to improve high voltage stability. Different PCB materials can be employed with thickness in the 0.4-1 mm range, and the hole spacing (pitch) in the 0.7-1.2 mm 

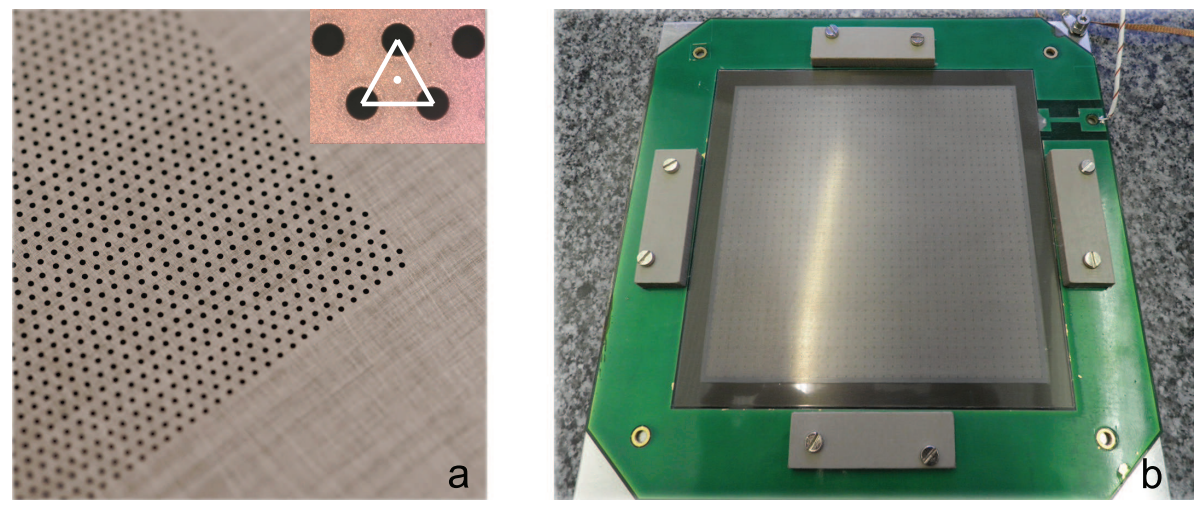

Figure 1: a) An example of THGEM detector, the holes are drilled using standard PCB production techniques, on top the equilateral triangle defined by 3 hole centres. b) A small size prototype of a bulk MicroMegas detector. The pillars of $\approx 300 \mu \mathrm{m}$ diameter and the mesh are visible.

range (see Fig. 1 a); The working principle is identical to the GEM one. The electrons driven to the holes region experience the strong electric field in the $50-70 \mathrm{kV} / \mathrm{cm}$ range, obtained by the voltage biasing of the two electrodes. These multipliers present specific features: the electrons collection and transport between cascaded elements is more effective than in GEMs, since the THGEM hole diameter is larger than the electron diffusion range, near the next hole region. In principle, THGEMs of large size can be produced in large series thanks to the multi- spindle high-frequency drilling heads that reduce the total drilling time and the corresponding cost: 1Euro/1000holes.

THGEMs have intrinsic mechanical stiffness and they are robust against damages produced by discharges. Due to the enlarged geometrical parameters, ten times GEM's ones, THGEMs offer spatial resolution in the $0.3-1 \mathrm{~mm}$ range. The reduced gaps between the multiplication stages, when multiple staked architecture is used, allow for their use in presence of magnetic fields.

THGEMs are produced by different companies, at affordable cost . Despite the easy production, the quality control and procedures applied to the standard PCB industrial manufacturing are not adequate for THGEMs.

Very recently a post-production protocol has been defined: a set of pre-selection material steps followed by a dedicated surface treatment and epoxy curing procedures have enabled the production of large size THGEMs reaching the expected detector performance and response[9].

Also for MicroMegas the recent Bulk technology introduction[11] has allowed the production of large size MicroMegas detectors $\left(\approx 1 \mathrm{~m}^{2}\right)$. The MicroMegas (Fig. 1, b) is a thin parallel plate avalanche counter. The multiplication stage is an extremely thin region (typically around $100 \mu \mathrm{m}$ ) between a thin metal grid, the micromesh, and the readout plane formed by pads or strip on a standard PCB frame. In the present state-of-the-art, regularly spaced ( $2 \mathrm{~mm}$ pitch) insulating pillars ( $200 \mu \mathrm{m}$ diameter) act as support keeping the gap between the anode and the mesh uniform at the price of limited localized efficiency loss. The mesh and the anodic read-out plane are biased so to obtain an electric field typically in the range 50 and $70 \mathrm{kV} / \mathrm{cm}$. Above the micromesh, a conversion region, a few mm thick, allows the formation of the primary ionization; a mild electric field (of the order of $1 \mathrm{kV} / \mathrm{cm}$ ) guides the electrons to the multiplication volume. The large difference of the electric fields above and below the micromesh shapes the electric field lines so to compress them 


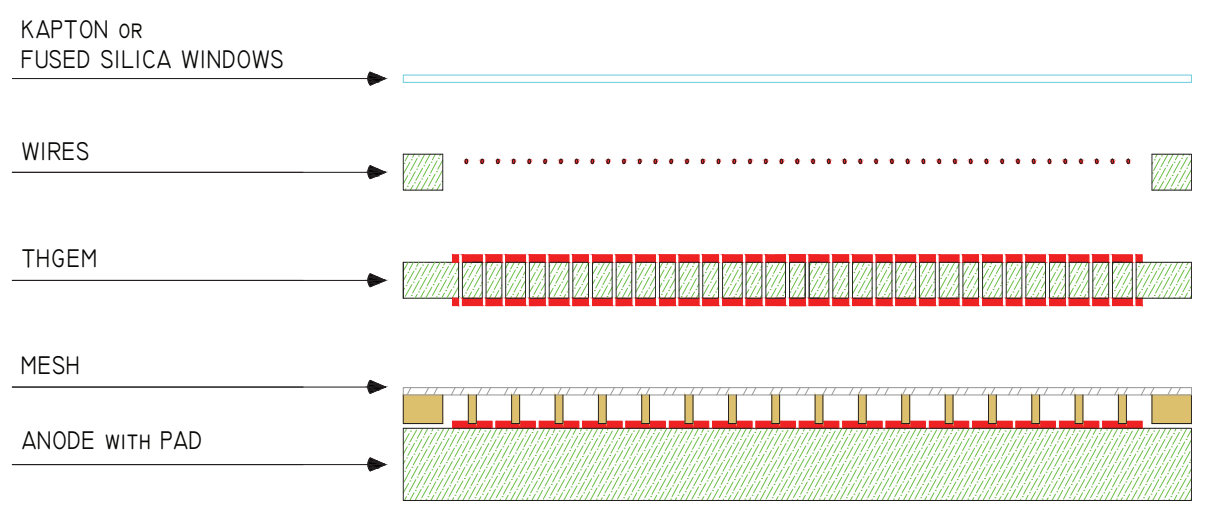

Figure 2: Sketch of the typical structure of the Hybrid detector (not to scale). The CsI is deposited on the top surface of the THGEM layer. The main elements: THGEM(s), the Bulk MicroMegas, and the Drift plane are visible.

from the conversion region into the mesh openings, providing almost complete transparency to the incoming electrons. Moreover, the majority of the field lines in the multiplication region end at the micromesh electrode ensuring very good trapping of the ions generated in the multiplication. The strong electric field, combined with the reduced gap, results in fast positive ions removal, preventing from space charge accumulation and, thanks to the reduced gap, the induced signals are very fast $(\approx 100 \mathrm{~ns})$ even if largely generated by the ion movement. The transverse size of the electron avalanche due to diffusion is in the $15 \mu \mathrm{m}$ range.

\section{Hybrid MPGD architectures.}

THGEMs have been extensively studied [5] and the results of a broad campaign of tests performed to characterize and evaluate their performance have been presented and discussed in $[13,14,15,16,17,18]$. The reader is referred to the cited publications and the reference therein for further details as well as for the optimisation of the THGEM parameters, gas mixtures and field configuration for single photon detection; MicroMegas detectors have been successfully employed in several apparatus and this technology has been proven reliable[19].

In the following the novel Hybrid Detector (HD) scheme, sketched in Fig. 2, obtained by the coupling of a MicroMegas detector and one or two THGEMs will be illustrated within the optimisation tests and the preliminary results obtained. The HD develops the concept of hybrid structures introduced to limit the spark rate in high rate experiments[19, 10], here the GEM foils are replaced by the more robust THGEM boards which are adequate for higher gain figures.

The minimal version of the HD is made by a Bulk-MM and a single THGEM stage, as illustrated in Fig. 2, but it can be equipped with a second THGEM when larger gain figures are required. The first THGEM acts as physical support where the CsI layer, the UV photon-converter, is deposited as well as electron amplification stage. It is optimized, in terms of geometrical parameters and operating voltages, to guarantee the highest photoelectron extraction and collection efficiency[17, 18]. The second THGEM, if present, allows to increment the maximum gain achievable with respect to the configuration with one THGEM only, it acts in fact both as electron multi- 


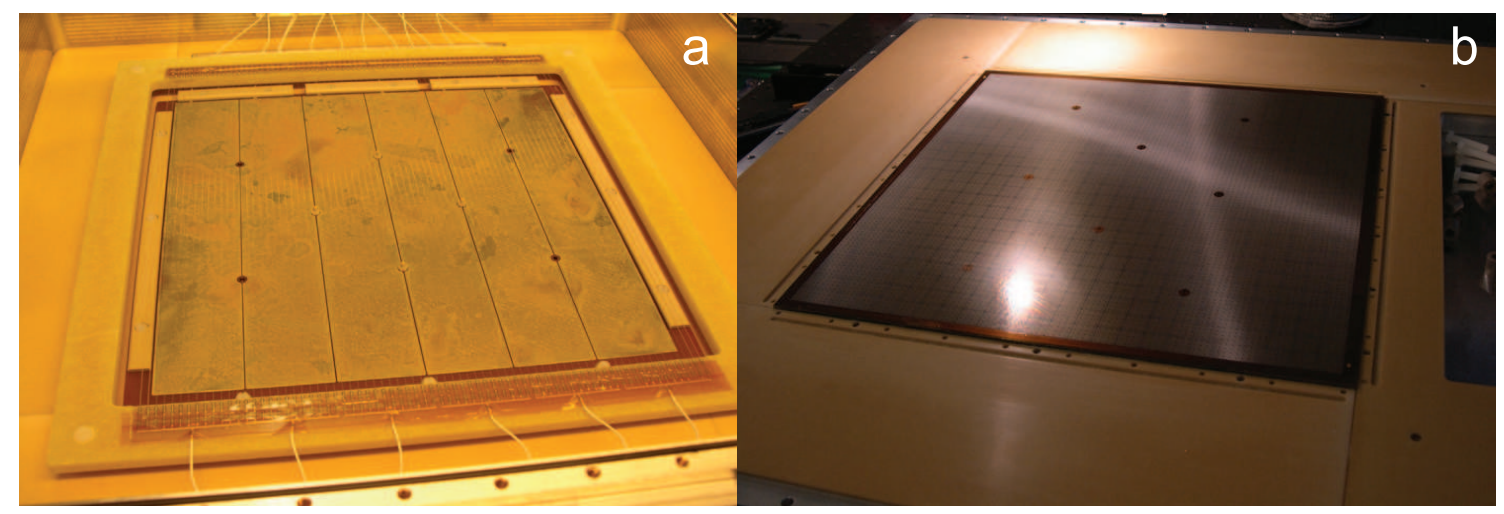

Figure 3: a) The assembled detector view: the THGEM segmentation into sectors and the the $100 \mu \mathrm{m}$ wire plane are visible. b) The $300 \times 300 \mathrm{~mm}^{2}$ bulk MicroMegas mesh glued on its support frame before the THGEM installation.

plier and as charge splitter, reducing the probability of exceeding locally in the holes, the maximum charge density, namely the Raether limit. This function is obtained geometrically arranging the two THGEM stages with the hole centres of the first THGEM aligned with the centres of the equilateral triangles of the second one, see Fig. 1 a). The THGEM(s) used have dimensions of $300 \mathrm{~mm}$ x $300 \mathrm{~mm}$ and are segmented into 6 sectors $300 \mathrm{~mm}$ x $50 \mathrm{~mm}$, splitting the accumulated charge between the sectors thus avoiding detector damages in case a discharge occurs (see Fig. 3 a)). The THGEM(s) geometrical parameters are: $0.4 \mathrm{~mm}$ holes diameter, $0.8 \mathrm{~mm}$ pitch and $0.4 \mathrm{~mm}$ PCB thickness. No rim, the clearance circular region around the THGEM hole, is present[13].

The electric field above the CsI coated THGEM, the Drift field, is defined by $100 \mu \mathrm{m}$ diameter wires with $2 \mathrm{~mm}$ pitch, placed at $20 \mathrm{~mm}$ distance from the top THGEM face, while the electric field between the THGEMs and the last THGEM and the mesh are named transfer fields (Fig. 2). The last stage of the hybrid detector makes use of a Bulk MM produced at CERN. The micromeshanode distance is $128 \mu \mathrm{m}$, the pillars, built to guarantee the mesh to anode distance constant all over the surface, have a diameter of $300 \mu \mathrm{m}$ and a pitch of $2 \mathrm{~mm}$ respectively, see Fig. $3 \mathrm{~b}$ ). The micromesh is a stainless steel grid of $18 \mu \mathrm{m}$ diameter woven wires separated by a distance of $\approx$ $53 \mu \mathrm{m}$. The anodic plane is segmented into 576 pads $12 \mathrm{~mm} \times 12 \mathrm{~mm}$ each.

The HD scheme presents several appealing features, the pre amplification generated by the THGEM reduces the inefficiency due to the pillars at the MicroMegas level and the transverse size of the electron distribution after the THGEM pre amplification may generate several avalanche processes at the MicroMegas stage: when this charge is collected by the same sensitive electrode, a single pad, it results in a larger effective gain. This principle can be enhanced adding a second THGEM stage and staggering the holes alignment in the two THGEM boards as previously mentioned.

An important characteristics of the MicroMegas stage is the strong reduction of the Ion Back Flow (IBF), provided the ratio of the field below and above the micromesh to be larger than 20, by trapping the ions at the micromesh[4]: large ion accumulation is avoided and a stable and fast detector operation is possible, this feature is very relevant for those applications where the IBF control is a strong requirement, as it is the case for gaseous photon detectors. 


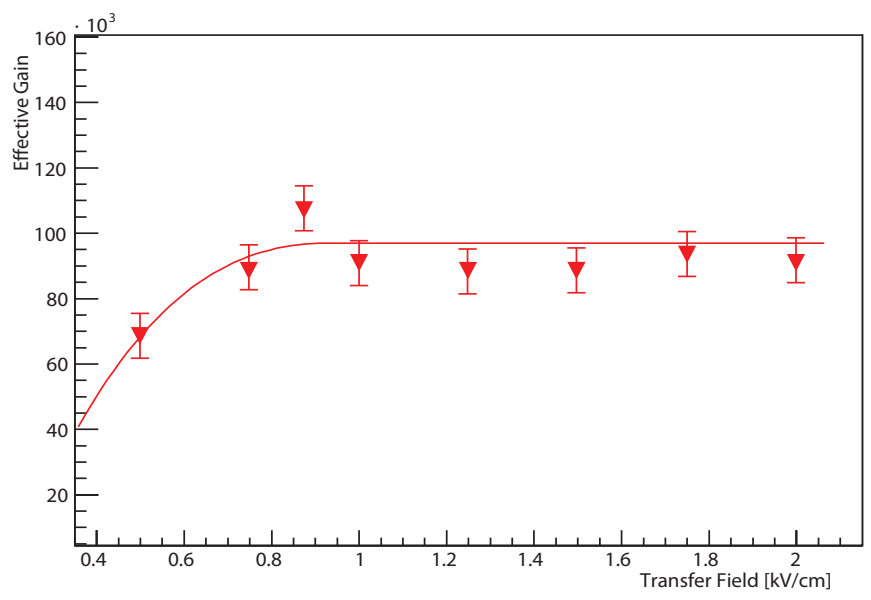

Figure 4: Effective gain variation as function of the transfer field, a value of $0.8 \mathrm{kV} / \mathrm{cm}$ guarantees full charge collection.

\section{Hybrid detector characterisation and performance tests.}

In this section the performance tests of the HD MicroMegas and of the full detector, (one THGEM and the Bulk MicroMegas) as well as their results will be illustrated. All the test sets are based on the collection of the amplitude signals generated by the $5.9 \mathrm{keV} \mathrm{X}$-Ray conversion in Ar emitted by a ${ }^{55} \mathrm{Fe}$ source. Signals are read at the $300 \mathrm{~mm}$ x $300 \mathrm{~mm}$ anode level, segmented into $57612 \mathrm{~mm}$ x $12 \mathrm{~mm}$ pads; the pads are electrically connected at groups of 16, segmenting the whole sensitive surface in 36 equally distributed independent sample points. The signals are then processed by a read-out chain composed by a CREMAT CR $110^{1}$ charge sensitive pre-amplifier, an Ortec $672^{2}$ amplifier and an MCA8000A digitizer by Amptek ${ }^{3}$.

At first the MicroMegas gain uniformity has been analysed, by measuring the effective gain at the 36 different sampling positions, while keeping constant the negative voltage applied on the micromesh. The gain non-uniformity measured as $\left(G_{\text {Max }}-G_{\text {Min }}\right) / G_{\text {Max }}$ at gain values of $10^{3}$ corresponds to $10 \%$, where $G_{M a x}$ and $G_{M i n}$ are the highest and lowes gain values measured.

The second step, after the THGEM mounting and biasing, consists in the optimization of the electric field between the THGEM bottom electrode and the micromesh $\left(E_{T R}\right)$. The field has been varied while both the micromesh and THGEM biasing voltage are kept constant. The corresponding effective gain is then measured. As illustrated in Fig. 4 values of $E_{T R}$ larger than $0.8 \mathrm{kV} / \mathrm{cm}$ guarantee the full charge collection from the THGEM pre-amplification stage while at lower voltages the electron charge is trapped at the THGEM level. The plateaux region extends up to $2 \mathrm{kV} / \mathrm{cm}$ as shown by the full line drawn as a guide line for the reader ${ }^{4}$.

The next step consists in optimizing the gain sharing between the THGEM and the MicroMegas

\footnotetext{
${ }^{1}$ Cremat, Inc., Watertown, Massachusset, USA

${ }^{2}$ ORTEC Advanced Measurement Technology, Inc, Oak Ridge, Tennessee, USA

${ }^{3}$ Amptek Inc., Bedford, Massachusset, USA

${ }^{4}$ It is not a fit
} 

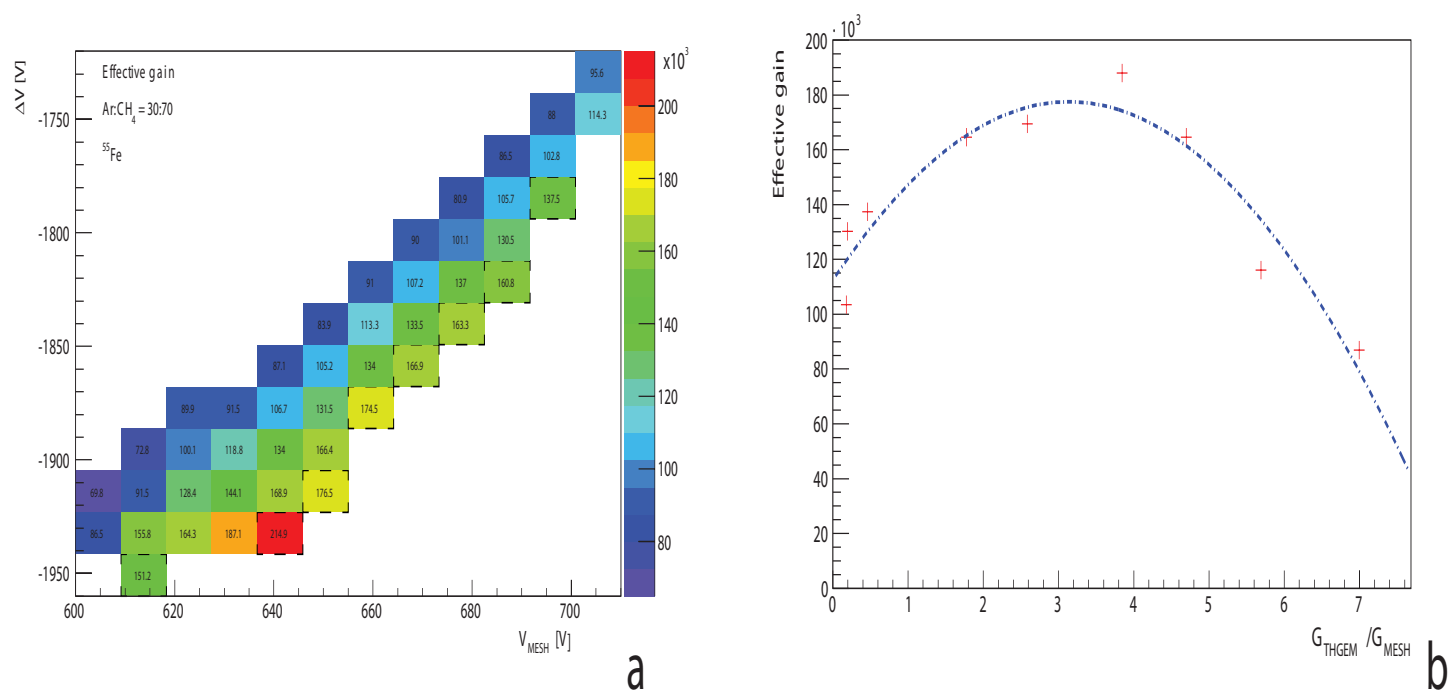

Figure 5: a): gain values of the hybrid detector as function of the THGEM $\Delta \mathrm{V}$ and of the mesh voltage. The square dotted boxes correspond to non stable detector operation. b): gain values of the hybrid detector as function of the THGEM and MicroMegas gain ratio obtained from the previous plot using the stable detector configuration data set.

stage: the total HD gain is given by $G_{T o t}=G_{M E S H} * G_{T H G E M}$ so similar values of $G_{T o t}$ can be reached in different gain sharing conditions of the THGEM and the MicroMegas. To study the effect of the sharing behaviour as well as to spot the most stable working configuration of the HD $G_{T o t}$ has been measured as function of the THGEM biasing voltage $(\Delta \mathrm{V})$ and of the mesh voltage. In figure 5 a) the gain values of the HD as function of the THGEM $\Delta V$ and of the mesh voltage ere plotted. The data points in squared dotted boxes are obtained while operating the detector in a unstable configuration, namely with an average time interval of 15 minutes between two occurrences of a discharge at the THGEM or at the MicroMegas level. For the remaining gain sharing configuration no discharges have been detected for a time interval of $\approx 1$ hour.

Using the values of the THGEM $\Delta V$ and of the micromesh voltages for which the detector is stably operating, the effective gain can be plotted as function of the ratio of the THGEM and MicroMegas gains $G_{T H G E M} / G_{M E S H}$. As illustrated by the dotted line in Fig. $5 \mathrm{~b}$ ), where the line is drawn as a guide line for the reader, ${ }^{5}$ the highest stable gain configuration is obtained for $\approx G_{T H G E M}=4 \cdot G_{M E S H}$.

The last step consists in studying the HD performance for different $\mathrm{CH}_{4}: \mathrm{Ar}$ gas mixtures with the aim to characterize the detector stability response as well as the effect the maximum gain reachable as function of the $\mathrm{CH}_{4}$ fraction of the gas mixture. The HD as single photon detector must in fact be operated in a Methane rich atmosphere $\left(\mathrm{CH}_{4} \geq 30 \%\right)$ in order to grant good photo electron extraction efficiency from the CsI layer[18]: the detector effective gain is measured as function of the THGEM $\Delta \mathrm{V}$ for the $-690 \mathrm{~V}$ fixed mesh voltage for different $\mathrm{Ar}: \mathrm{CH}_{4}$ ratios. Fig. 6 shows the measurement result of the effective gain for two different gas mixtures: $\mathrm{Ar}: \mathrm{CH}_{4}$ 30:70 (left) and

\footnotetext{
$5_{\text {it is not a fit. }}$
} 

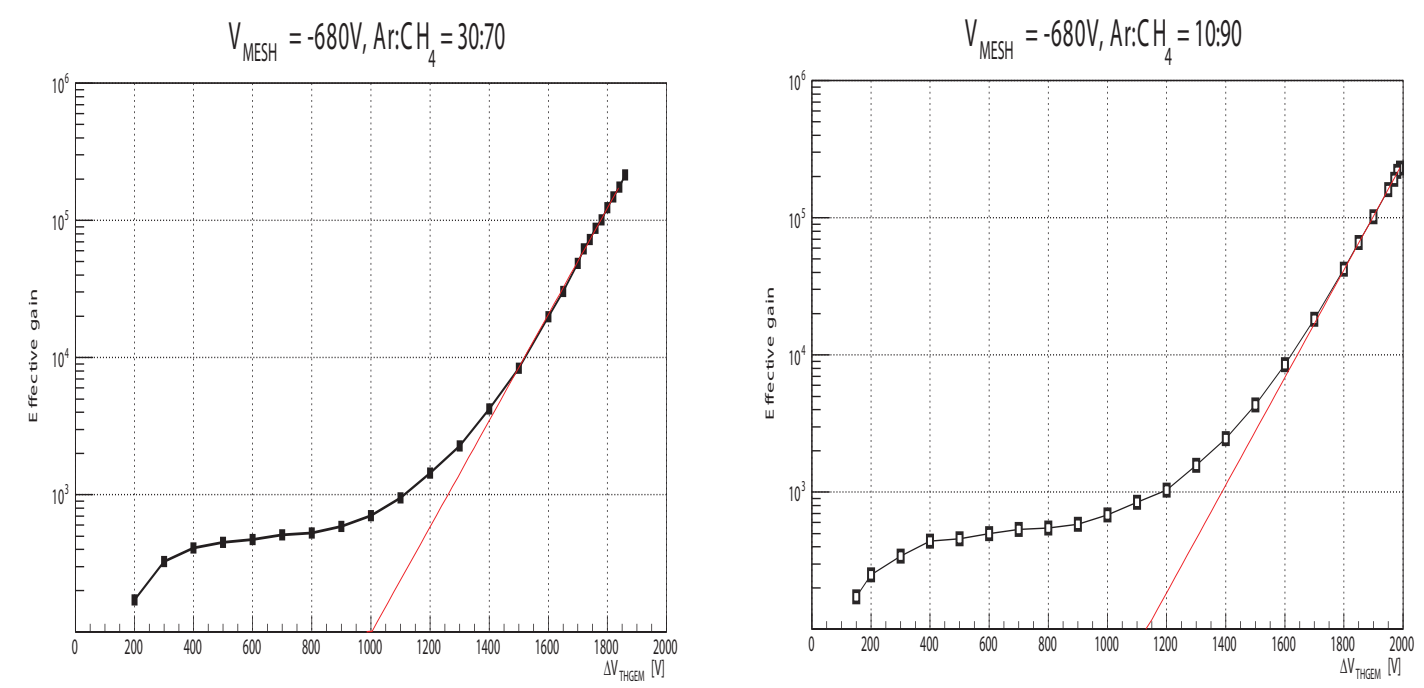

Figure 6: Left: effective gain as function of the THGEM biasing voltage for the $-690 \mathrm{~V}$ fixed mesh voltage for $\mathrm{Ar}: \mathrm{CH}_{4}$ 30:70 mixture. Right: effective gain is as function of the THGEM biasing voltage for the -690 $\mathrm{V}$ fixed mesh voltage for $\mathrm{Ar}: \mathrm{CH}_{4}$ 10:90 mixture.

Ar: $\mathrm{CH}_{4}$ 10:90 (right).

The plot illustrates how at voltages lower than $-200 \mathrm{~V}$ the THGEM is not transparent to electrons from the ${ }^{55} \mathrm{Fe} \mathrm{X}$-ray conversion: the effective gain is low $\approx 0$ since the primary ionization does not undergo multiplication: the focusing electric field generated by the THGEM biasing voltage is not enough to drive the electrons inside the holes volume despite the presence of $1.5 \mathrm{kV} / \mathrm{cm}$ Drift field. Increasing the THGEM $\Delta V$ the electron collection becomes more and more efficient: between $400 \mathrm{~V}$ and $800 \mathrm{~V}$ of $\Delta V$ the THGEM is transparent to the electrons, its gain is approximately unitary and the total measured gain of the detector $(\approx 600)$ is mostly due to the MicroMegas stage. Increasing more the $\Delta V$ voltage, above $1 \mathrm{kV}$ the HD gain increases exponentially: the THGEM electric field inside the holes is large enough to contribute to the electron multiplication. The maximum gain achieved is $\approx 2 \times 10^{5}$.

Comparing Fig. 6 left and right where the $\mathrm{CH}_{4}$ fraction is increased from 50\% to $90 \%$ it can be noticed that the maximum gain achievable remains unchanged but it is reached for a larger total value of the applied voltage of nearly $120 \mathrm{~V}$. A mild slope increase is observed too when moving from lower towards a larger $\mathrm{CH}_{4}$ fraction. For the other $\mathrm{Ar}: \mathrm{CH}_{4}$ mixture the detector performance remain unchanged.

The complete detector uniformity response is checked by measuring the gain response as function of the THGEM biasing voltage for each of the 6 independent sectors. Fig. 7 top shows an example of a spectrum collected using the ${ }^{55} \mathrm{Fe}$ source in $\mathrm{Ar} / \mathrm{CH}_{4}$ 30:70. The voltage applied to the mesh electrode is $V_{\text {mesh }}=0.55 \mathrm{kV}$, the field between the micromesh and the THGEM is $0.9 \mathrm{kV} / \mathrm{cm}$ and the bias voltage across the THGEM layers is $\Delta \mathrm{V}=1.2 \mathrm{kV}$, the field above the THGEM is $1.5 \mathrm{kV} / \mathrm{cm}$; the measured effective gain is $1.5 \times 10^{3}$. The main peak as well as the escape peak are clearly identified.

The full detector has been tested to check the performance of the 6 sectors as illustrated in 

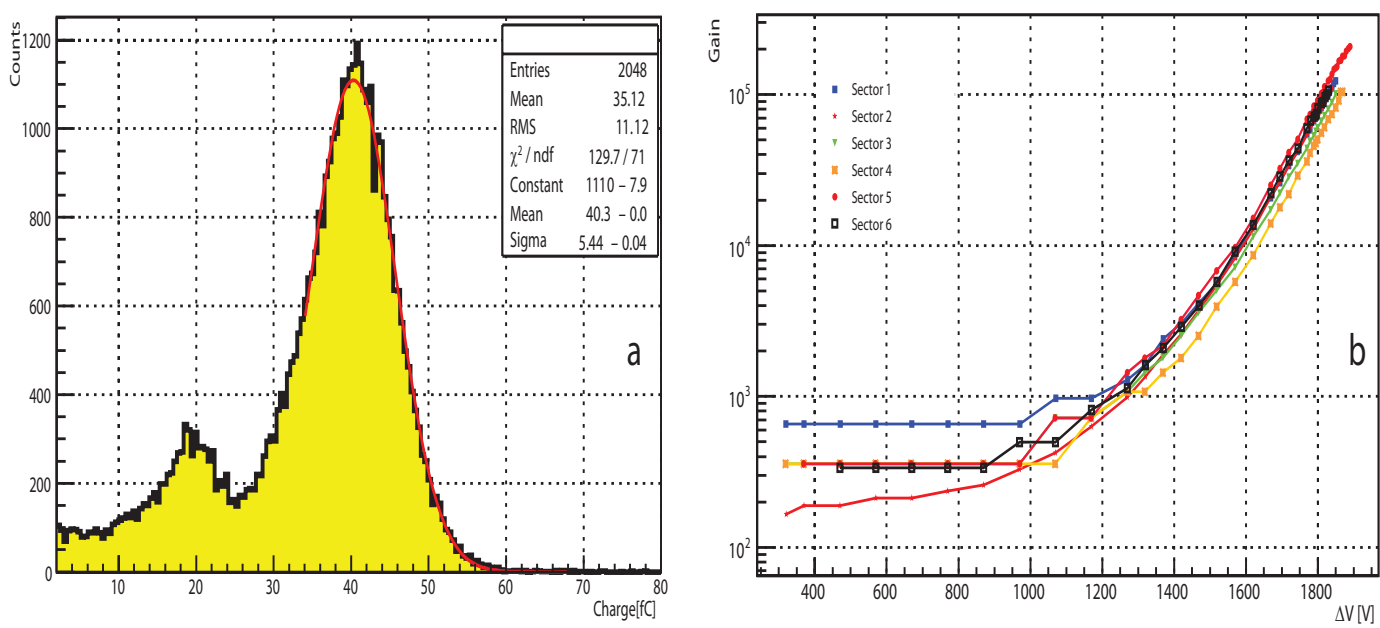

Figure 7: Amplitude spectra obtained with the hybrid detector prototype using the ${ }^{55} \mathrm{Fe}$ source in in $\mathrm{Ar}: \mathrm{CH}_{4}$ 30:70 mixture; estimated gain: $1.3 \times 10^{3}$. Right: gain as function of $\Delta \mathrm{V}$ for $V_{\text {mesh }}=640 \mathrm{~V}$, for each of the the six THGEM Sectors

Fig. 7 where for $0.64 \mathrm{kV}$ fixed voltage of the mesh the gain of the detector is measured varying the $\Delta V$ across the THGEM multiplier. As already mentioned before, up to $1 \mathrm{kV} \Delta V$, the whole multiplication takes mostly place in the MM stage. The effective gain data points for each of the 6 sectors are compatible within the experimental error of $\approx 10 \%$, confirming the good detector uniformity response. For all the sector the gain of $1.2 \times 10^{5}$ is reachable.

It is important to remark that the hybrid detector can also profit from the use of a resistive anode[20] with two major advantages: the sensitive readout electronics is decoupled from the direct current during occasional discharges, protecting it; the spark energy is reduced and the deadtime due to the detector discharges is almost completely suppressed, introducing the possibility to operate in high rate environments[20].

\section{Conclusions}

We report about the R\&D studies of the hybrid MPGD-based architecture of single photon detector. In particular from the preliminary results the hybrid architecture exhibit extremely good performance in terms of gain stability. Further tests are needed to check the long term operation as well as the behaviour of larger-size photon detectors. Two hybrid $300 \mathrm{~mm}$ x $300 \mathrm{~mm}$ detectors equipped with CsI coated THGEMs and fused silica radiator for Cherenkov light generation will undergo a full campaign of test at the T10 beam line at CERN during the late summer 2014 to prove the reliable operation in real experimental environment conditions. 


\section{References}

[1] A. Oed, Nucl. Instr. and Meth. A 263 (1988) 351

[2] F. Sauli, Nucl. Instr. and Meth. A 386 (1997) 531

[3] F. Sauli, Nucl. Instr. and Meth., A 580 (2007) 971 and refs. therein

[4] Y. Giomataris et al., Nucl. Instr. and Meth. A 376 (1996) 29.

[5] A. Breskin et al., Nucl. Instr. and Meth. A 598 (2009) 107 and refs. therein.

[6] J.M. Bidault et al., Nucl. Phys. B 158 (Proc. Suppl.) (2006) 199; R. Oliveira et al., /http://arxiv.org/abs/physics/0701154S; G. Agocs et al., JINST 3 (2008) P02012; G. Agocs et al., Nucl.Instr. and Meth. A 595 (2008) 128; A. Di Mauro et al., Nucl. Instr. and Meth. A 581 (2007) 225. J.F.C.A. Veloso et al., Rev.Sc. Instr. 71 (2000) 2371; A.V. Lyashenko et al., JINST 2 (2007) P08004.

[7] 10. A.V. Lyashenko et al., Nucl. Instr. and Meth. A 598 (2009) 116

[8] 11. A. Ochi et al., Nucl. Instr. and Meth. A 471 (2001) 264.

[9] M Alexeev et al. 2014 JINST 9 C03046

[10] CLAS12 Technical Design Report V5.1, JLAB (2008).

[11] J. Bouchez et al., Nucl. Instr. and Meth. A 574 (2007) 425 and refs. therein

[12] R. Chechik et al., Nucl. Instr. and Meth. A 535 (2004) 303.

[13] M. Alexeev et al., Nucl. Instrum. Meth. A 610 (2009) 174.

[14] M. Alexeev et al., Nucl. Instrum. Meth. A 617 (2010) 396.

[15] M. Alexeev et al., 2010 JINST 5 P03009.

[16] M. Alexeev et al., Nucl. Instrum. Meth. A 639 (2011) 130.

[17] M. Alexeev et al., Nucl. Instrum. Meth. A 623 (2010) 129.

[18] M. Alexeev et al., Physics Procedia 37 (2012) 781.

[19] P. Abbon et al. , Nucl. Instr. Meth. A 577 (2007) 455.

[20] Manjarres, et al., 2012 JINST 7 C03040 